\title{
Darstellung von 4-Dinitroindigo
}

\author{
von \\ Julius Schwarz.
}

Aus dem Laboratorium für chemische Technologie organischer Stoffe an der k. k. technischen Hochschule in Wien.

(Vorgelegt in der Sitzung am 13. Juli 1905.)

Adolf v. Baeyer hat bereits im Jahre 1879 einen Dinitroindigo hergestellt, ${ }^{1}$ den er damals noch $»$ Nitroindigo " nannte, da die Molekulargröße des Indigos erst später von ihm festgestellt wurde.

Zur Darstellung dieses Dinitroindigos schlug Baeyer folgenden Weg ein: Er nitrierte zunächst das Isatin, welches in konzentrierter Schwefelsäure gelöst wurde, mit Salpeter, verwandelte das so erhaltene Nitroisatin mit Phosphorpentachlorid und Phosphoroxychlorid in das Chlorid und führte dieses in den entsprechenden Dinitroindigo über, indem er es mittels einer Auflösung von Jodwasserstoffsäure in Eisessig reduzierte. Es war dieser Vorgang ein Abbau und dann ein Aufbau, da Baeyer das Isatin als Spaltungsprodukt des Indigos, nämlich durch Oxydation desselben mittels Salpetersäure, erhielt.

Ich versuchte nun, einen Dinitroindigo direkt, ausgehend von einer Nitroaminobenzoesäure, aufzubauen.

\section{Darstellung der $m$-Nitrophenylglyzin-o-Carbonsäure.}

$\mathrm{Zu}$ diesem $Z$ weck acetylierte ich nach Nölting und Collin ${ }^{2} p$-Nitro-o-Toluidin mit Essigsäureanhydrid, oxydierte das erhaltene Acet- $p$-Nitro-o-Toluid nach der Arbeit von

1 Berl. Ber., 12, 1316.

2 Berl. Ber., 17, 269. 
Wheeler und Barnes ${ }^{1}$ mit Kaliumpermanganat zu Acet4-Nitro-2-Aminobenzoesäure und führte diese durch Absprengung der Acetylgruppe ${ }^{2}$ in 4-Nitro-2-Aminobenzoesäure über. Die durch Umkristallisation gereinigte 4-Nitro-2-Aminobenzoesäure wurde nun, analog der Vorschrift von Mauthner und Suida ${ }^{3}$ für die Herstellung der Phenylglyzin-o-Carbonsäure, mit Monochloressigsäure zu kondensieren versucht.

1.Versuch. $5 g$ 4-Nitro-2-Aminobenzoesäure, $2 \cdot 8 \mathrm{~g}$ Monochloressigsäure und $5 \mathrm{~g}$ Soda wurden in $120 \mathrm{~cm}^{3}$ Wasser gelöst und 2 Stunden lang am Rückflußkühler gekocht. Es wurde hier sofort ein Überschuß an Monochloressigsäure und Soda genommen, damit nicht ein Teil der 4-Nitro-2-Aminobenzoesäure unangegriffen bleiben sollte, wie dies auch bei der Herstellung der Phenylglyzin-o-Carbonsäure notwendig ist. Nach dem Abkühlen wurde die alkalische Flüssigkeit vorsichtig mit konzentrierter Salzsäure versetzt und der erhaltene gelbe Niederschlag abgesaugt. Dieser wurde hierauf durch Umkristallisieren aus heißem Wasser gereinigt. Ich erhielt gelbe bis orange gefärbte Nadeln vom Schmelzpunkte $230^{\circ}$, die bei $105^{\circ}$ getrocknet wurden und bei der Analyse folgende Resultate lieferten:

$0 \cdot 2052 g$ Substanz gaben $0 \cdot 3444 g$ Kohlensäure und $0 \cdot 0642 g$ Wasser.

$0.2290 \mathrm{~g}$ Substanz gaben $25 \cdot 4 \mathrm{~cm}^{3}$ Stickstoff bei einer Temperatur von $11^{\circ} \mathrm{C}$. und $752 \mathrm{~mm}$ Barometerstand.

In 100 Teilen:

\begin{tabular}{|c|c|c|c|}
\hline & $\begin{array}{r}\text { Berechnet für } \\
\mathrm{C}_{9} \mathrm{H}_{8} \mathrm{O}_{6} \mathrm{~N}_{2}\end{array}$ & $\begin{array}{r}\text { Berechnet für } \\
\mathrm{C}_{7} \mathrm{H}_{6} \mathrm{O}_{4} \mathrm{~N}_{2}\end{array}$ & Gefunden \\
\hline$C \ldots$ & $45 \cdot 00$ & $46 \cdot 15$ & $45 \cdot 77$ \\
\hline $\mathrm{H}$ & $3 \cdot 33$ & $3 \cdot 30$ & $3 \cdot 47$ \\
\hline N... & $11 \cdot 67$ & $15 \cdot 38$ & $13 \cdot 09$ \\
\hline
\end{tabular}

1 American chemical Journal, 20, 219.

2 American chemical Journal, 20, 221.

3 Monatshefte für Chemie, 9 (1888), 728. 
Das erhaltene Produkt enthielt also zirka $40 \% \mathrm{~m}$-Nitrophenylglyzin-o-Carbonsäure und $60 \%$ unveränderte 4 -Nitro2-Aminobenzoesäure. Ich versuchte nun eine Trennung der beiden Körper durch fraktionierte Kristallisation aus heißem Wasser vorzunehmen, doch gelang mir dies nicht, da beide ziemlich gleiche Löslichkeit zeigten.

2. Versuch. Ich war nun bestrebt, ein reineres Produkt dadurch zu erhalten, daß ich den Überschuß an Monochloressigsäure erhöhte, was natürlich auch eine Vergrößerung der Sodamenge zur Folge hatte, und daß ich die Einwirkungsdauer der beiden Komponenten durch längeres Kochen erhöhte.

Es wurden nun $5 \mathrm{~g}$ 4-Nitro-2-Aminobenzoesäure, $5.1 \mathrm{~g}$ Monochloressigsäure und $10 \mathrm{~g}$ Soda in $150 \mathrm{~cm}^{3}$ Wasser gelöst und 8 Stunden lang am Rückflußkühler gekocht. Genau auf dieselbe Art wie beim ersten Versuche wurde die $m$-Nitrophenylglyzin-o-Carbonsäure gewonnen und wieder aus Wasser umkristallisiert. Die erhaltenen Nadeln vom Schmelzpunkte $235^{\circ}$ waren dunkelgelb; sie wurden bei $105^{\circ}$ getrocknet und lieferten bei der Analyse folgende Resultate:

$0.1518 g$ Substanz gaben $0 \cdot 2520 g$ Kohlensäure und $0.0473 g$ Wasser.

$0.1986 \mathrm{~g}$ Substanz gaben $21.2 \mathrm{~cm}^{3}$ Stickstoff bei $10^{\circ} \mathrm{C}$. und $748 \mathrm{~mm}$ Barometerstand.

In 100 Teilen:

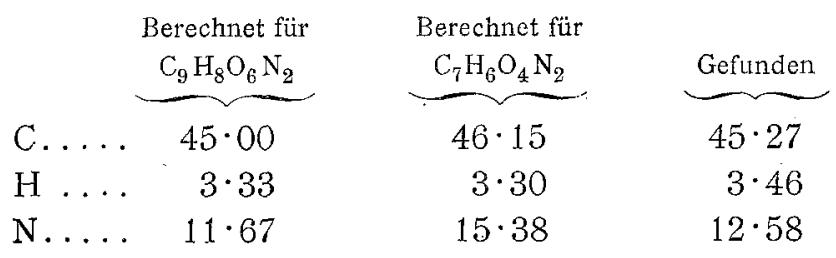

Es waren also nur mehr zirka $20 \%$ der 4-Nitro-2-Aminobenzoesäure unangegriffen.

3. Versuch. Ich vergrößerte daher wieder den Überschuß an Monochloressigsäure und Soda, so daß ich folgende Mengenverhältnisse anwandte: $5 g$ 4-Nitro-2-Aminobenzoesäure, $10 \cdot 5 \mathrm{~g}$ Monochloressigsäure und $13.5 \mathrm{~g}$ Soda. Diese 
wurden in $150 \mathrm{~cm}^{3}$ Wasser gelöst und 8 Stunden lang am Rückflußkühler gekocht, hierauf erkalten gelassen und die $m$-Nitrophenylglyzin-o-Carbonsäure aus der alkalischen Flüssigkeit mit konzentrierter Salzsäure gefällt. Der hellgelbe voluminöse Niederschlag wurde abfiltriert und durch Umkristallisation aus heißem Wasser gereinigt. Man erhält auf diese Weise hellgelbe, sehr feine und lange Nadeln, die einen schwach säuerlichen Geschmack besitzen und bei 240 bis $242^{\circ}$ unter Zersetzung schmelzen, wobei jedoch schon von $225^{\circ}$ an eine immer mehr fortschreitende Schwärzung der Substanz im Schmelzpunktsröhrchen wahrzunehmen ist. Die bei $105^{\circ}$ getrocknete Säure ergab bei der Analyse folgende Resultate:

$0.1369 \mathrm{~g}$ Substanz lieferten bei der Verbrennung $0.2265 \mathrm{~g}$ Kohlensäure und $0 \cdot 0423 \mathrm{~g}$ Wasser.

$0 \cdot 1979 \mathrm{~g}$ Substanz ergaben $20 \cdot 2 \mathrm{~cm}^{3}$ Stickstoff bei einer Temperatur von $11^{\circ} \mathrm{C}$. und $740 \mathrm{~mm}$ Barometerstand.

In 100 Teilen:

\begin{tabular}{|c|c|c|}
\hline & $\begin{array}{c}\text { Berechnet für } \\
\mathrm{C}_{9} \mathrm{H}_{8} \mathrm{O}_{6} \mathrm{~N}_{2}\end{array}$ & Gefunden \\
\hline $\mathrm{C}$ & $45 \cdot 00$ & $44 \cdot 90$ \\
\hline & $3 \cdot 33$ & $3 \cdot 42$ \\
\hline $\mathrm{N} \ldots \ldots$ & $11 \cdot 67$ & $11 \cdot 95$ \\
\hline
\end{tabular}

Es war also dieses Produkt nun reine $m$-Nitrophenylglyzin-o-Carbonsäure. Während die Phenylglyzin-o-Carbonsäure beim Kochen mit verdünnten Säuren unter Kohlensäureabspaltung sich in Phenylamidoessigsäure verwandelt und daher aus Methylalkohol umkristallisiert werden muß, ${ }^{1}$ bleibt die 4-Nitrophenylglyzin-o-Carbonsäure bei Vornahme dieser Operation unverändert und kann daher aus Wasser umkristallisiert werden. Die Ausbeute beträgt bei dieser Methode zirka $75 \%$ der theoretischen.

Die $m$-Nitrophenylglyzin-o-Carbonsäure ist in Alkohol sehr leicht, ziemlich leicht in Aceton, Petroläther und heißem

1 Beilstein, Supplement, II, p. 784. 
Wasser, schwerer in Äther, Benzol, Chloroform und kaltem Wasser löslich. Mit Eisenchloridlösung gibt die wässerige Lösung einen rotbraunen, flockigen Niederschlag. Kupferacetat, Silbernitrat, Bleiacetat sowie Quecksilberchlorid erzeugen keine Fällungen. Fehling'sche Lösung wird nicht reduziert. Im Glasröhrchen erhitzt, zersetzt sich die $m$-Nitrophenylglyzin-o-Carbonsäure bei teilweiser Sublimation.

\section{Herstellung des sauren Kalisalzes.}

Versetzt man die alkoholische Lösung der $m$-Nitrophenylglyzin-o-Carbonsäure mit alkoholischer Kalilauge, so fällt das Kaliumsalz als ein hellroter kristallinischer Niederschlag, der aus mikroskopisch kleinen Nadeln besteht, aus. Man filtriert $\mathrm{ab}$ und wäscht so lange mit Alkohol, bis das Filtrat keine alkalische Reaktion mehr zeigt. Das bei $105^{\circ}$ getrocknete Salz gab bei der Analyse folgendes Resultat:

$0 \cdot 4603 g$ des Salzes lieferten $0 \cdot 1468 g$ Kaliumsulfat.

In 100 Teilen:

$\mathrm{K} \ldots \ldots \ldots \underbrace{\begin{array}{c}\text { Berechnet für } \\ \mathrm{C}_{9} \mathrm{H}_{7} \mathrm{O}_{6} \mathrm{~N}_{2} \mathrm{~K}\end{array}}_{14 \cdot 03} \quad \underbrace{\text { Gefunden }}_{14 \cdot 31}$

Das saure Kaliumsalz der $m$-Nitrophenylglyzin-o-Carbonsäure ist in Wasser äußerst leicht löslich, fast unlöslich in allen organischen Lösungsmitteln. Es gibt mit Kupferacetat in der Wärme einen rotbraunen Niederschlag, was auf eine Reduktion schließen läßt.

Silbersa1z. Mit Silbernitrat gibt das saure Kaliumsalz einen orangegelben voluminösen Niederschlag, der sich am Licht immer dunkler rot färbt und sich schließlich unter Silberausscheidung schwärzt und zersetzt. Der gelbe Niederschlag löst sich sowohl in Salpetersäure als auch in Ammoniak.

$0 \cdot 2083 \mathrm{~g}$ Silbersal $z$ wurden bei $105^{\circ}$ getrocknet und gaben bei der Analyse $0 \cdot 1082 g$ Silber. 
In 100 Teilen: Berechnet für

$$
\mathrm{Ag} \ldots \ldots . \underbrace{\mathrm{C}_{9} \mathrm{H}_{6} \mathrm{O}_{6} \mathrm{~N}_{2} \mathrm{Ag}_{2}}_{47 \cdot 58} \quad \frac{\text { Gefunden }}{51 \cdot 94}
$$

Darstellung der 5-Nitrophenyliminodiessigsäure-2-Carbonsäure.

Mumme erhielt die Phenyliminodiessigsäure-2-Carbonsäure ${ }^{1}$ durch Kochen von zwei Teilen Monochloressigsäure mit einem Teil Anthranilsäure in neutraler wässeriger Lösung oder durch Kochen der Natriumsalze dieser Säuren mit Wasser. Ich erhielt die 5-Nitrophenyliminodiessigsäure-2-Carbonsäure als Nebenprodukt bei der Herstellung der $m$-Nitrophenylglyzin-o Carbonsäure. Wenn man das stark salzsaure Filtrat, welches man nach dem Absaugen der $m$-Nitrophenylglyzin$o$-Carbonsäure erhält, einige Tage stehen läßt, so scheiden sich kleine, gelblichweiß gefärbte Nadeln aus. Diese wurden abfiltriert und da sie in allen organischen Lösungsmitteln äußerst leicht löslich sind und daher nicht umkristallisiert werden konnten, in möglichst wenig Wasser gelöst und mit einem bedeutenden Überschusse von konzentrierter Salzsäure versetzt. Anfangs fiel nichts aus; nach einigen Tagen jedoch erhielt ich große, feine, fast rein weiße Nadeln, welche sich bei $177^{\circ}$ unter Schwärzung zersetzen, ohne daß ein Schmelzen selbst bei höheren Temperaturen beóbachtet werden konnte. Die Analyse der im Vakuum getrockneten Säure ergab folgendes Resultat:

$0.1698 \mathrm{~g}$ Substanz lieferten bei der Verbrennung $0.2759 \mathrm{~g}$ Kohlensäure und $0.0520 \mathrm{~g}$ Wasser.

$0 \cdot 2439 \mathrm{~g}$ Substanz gaben $20 \cdot 2 \mathrm{~cm}^{3}$ Stickstoff bei $10^{\circ} \mathrm{C}$. und $748 \mathrm{~mm}$ Barometerstand.

\begin{tabular}{|c|c|c|}
\hline tenen. & $\begin{array}{l}\text { Berechnet für } \\
\mathrm{C}_{10} \mathrm{H}_{10} \mathrm{O}_{8} \mathrm{~N}_{2}\end{array}$ & Gefunden \\
\hline $\mathrm{C}$ & $44 \cdot 30$ & $44 \cdot 31$ \\
\hline H. . & $3 \cdot 36$ & $3 \cdot 40$ \\
\hline $\mathrm{N}$ & $9 \cdot 39$ & $9 \cdot 76$ \\
\hline
\end{tabular}

In 100 Teilen:

1 Berl. Ber, 33, 3182 . 
Alkoholische Kalilauge fällt aus der alkoholischen Lösung der 5 Nitrophenyliminodiessigsäure-2-Carbonsäure das Kalisalz derselben als hellgelben Niederschlag aus. Eisenchloridlösung gibt keine Fällung.

Behufs Überführung der $m$-Nitrophenylglyzin-o Carbonsäure in Dinitroindigo versuchte ich die Synthese von Karl Heumann, ${ }^{1}$ indem ich die Säure mit Ätzkali im Wasserstoffstrome zusammenschmolz. Doch gelangte ich zu keinem günstigen Resultat, da die Zersetzung der $m$-Nitrophenylglyzin-o-Carbonsäure bereits erfolgt, bevor noch die beiden Körper in geschmolzenem Zustand aufeirander einwirken können.

\section{Darstellung des 4-Nitrodiacetylindoxyls.}

Um nun zum Dinitroindigo auf einem andern Wege zu gelangen, versuchte ich die Kondensation der $m$-Nitrophenylglyzin-o-Carbonsäure mit Essigsäureanhydrid zu 4-Nitrodiacetylindoxyl analog der Herstellung von Diacetylindoxyl aus Phenylglyzin-o-Carbonsäure. ${ }^{2}$

$5 g$ trockenes und gepulvertes Natriumacetat wurden in $25 g$ Essigsäureanhydrid unter Erwärmung gelöst und hierauf $5 g m$-Nitrophenylglyzin-o-Carbonsäure in Pulverform nach und nach eingetragen. Die Bildung des 4-Nitrodiacetylindoxyls geht nach folgender Gleichung vor sich:

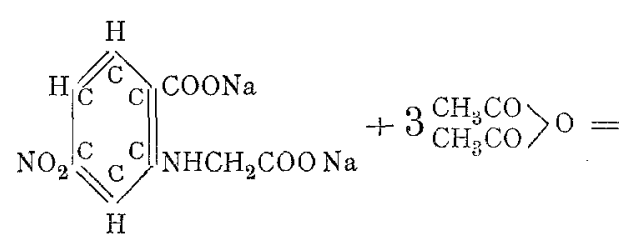

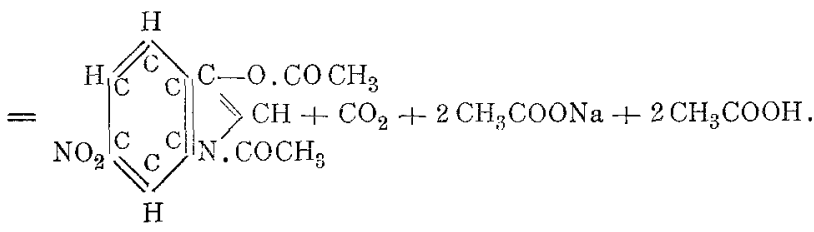

1 Berl. Ber., 23, 3431.

2 C. 1900 , II, 615. 
Nachdem man an dem Aufhören der Kohlensäureentwicklung das Ende der Reaktion erkannt hat, wird noch 15 Minuten lang am Rückflußkühler zum Kochen erhitzt und hierauf das überschüssige Essigsäureanhydrid am besten im Vakuum abdestilliert. Die zurückbleibende braune kristallinische Masse, bestehend aus Natriumacetat und 4-Nitrodiacetylindoxyl, wird mit heißem Wasser ausgelaugt und abfiltriert. Am Filter verbleibt das in Wasser fast unlösliche 4-Nitrodiacetylindoxyl, welches zur Reinigung aus Alkohol unter Zusatz von Tierkohle umkristallisiert wird. Das Umkristallisieren unter Zusatz von Tierkohle muß so lange wiederholt werden, bis man hellgelbe, große Nadeln vom Schmelzpunkte $190^{\circ}$ erhäit. Die Rohausbeute beträgt etwa $80 \%$ der theoretischen.

Die Analyse des bei $105^{\circ}$ getrockneten 4-Nitrodiacetylindoxyls ergab folgendes Resultat:

$0 \cdot 2454 g$ Substanz lieferten $0.4954 g$ Kohlensäure und $0 \cdot 0864 \mathrm{~g}$ Wasser.

$0.2032 \mathrm{~g}$ Substanz gaben $19 \mathrm{~cm}^{3}$ Stickstoff bei $15^{\circ} \mathrm{C}$. und einem Barometerstand von $748 \mathrm{~mm}$.

$0.2311 \mathrm{~g}$ Substanz verbrauchten bei der Acetylgruppenbestimmung nach Wenzel $16 \cdot 5 \mathrm{~cm}^{3} \mathrm{n} / 10$ Kalilauge.

In 100 Teilen:

\begin{tabular}{|c|c|c|}
\hline & $\begin{array}{l}\text { Berechnet für } \\
\mathrm{C}_{12} \mathrm{H}_{10} \mathrm{O}_{5} \mathrm{~N}_{2}\end{array}$ & Gefunden \\
\hline C. . & $55 \cdot 11$ & $55 \cdot 05$ \\
\hline $\mathrm{H}$ & $3 \cdot 81$ & $3 \cdot 91$ \\
\hline$N \ldots \ldots$ & $10 \cdot 68$ & $10 \cdot 89$ \\
\hline $\mathrm{CH}_{3} \mathrm{CO} \ldots$ & $32 \cdot 82$ & $30 \cdot 70$ \\
\hline
\end{tabular}

4-Nitrodiacetylindoxyl ist in kaltem und heißem Wasser fast unlöslich, ziemlich leicht in Alkohol, Äther, Aceton, Benzol und Ligroin. In kalter Sodalösung fast unlöslich, löst es sich darin beim Erwärmen mit dunkelgrüner Farbe auf. In konzentrierter Schwefelsäure ist es mit tief dunkelblauer Farbe löslich.

1 Monatshefte für Chemie, 18 (1897), 659. 


\section{Darstellung des 4-Dinitroindigos.}

Behufs Absprengung der Acetylgruppen und Oxydation des sich bildenden 4-Nitroindoxyls zu 4-Dinitroindigo versuchte ich die Verseifung sowohl mit Sodalösung als auch mit konzentrierter Salzsäure durchzuführen. Die erhaltenen Produkte, im ersten Falle grün, im zweiten schwarz, ergaben jedoch nach dem Umkristallisieren aus Alkohol unter Zusatz von Tierkohle das unveränderte 4-Nitrodiacetylindoxyl in gelben Nadeln zurück. Ich versuchte nun die Absprengung der Acetylgruppen mit Schwefelsäure vorzunehmen. $\mathrm{Zu}$ diesem Zwecke wurden $5 g^{-}$4-Nitrodiacetylindoxyl in konzentrierter Schwefelsäure unter Erwärmen gelöst. Die tief dunkelblaue Lösung wurde in viel Wasser gegossen und der dunkelblaue Niederschlag von 4-Dinitroindigo abfiltriert und so lange mit heißem Wasser gewaschen, bis das Filtrat keine saure Reaktion mehr zeigte. Der Niederschlag wurde getrocknet und hierauf mit reinem Anilin gekocht, in welchem er sich mit dunkelgrüner Farbe löste. Die so erhaltene Lösung wurde dann vorsichtig mit konzentrierter Salzsäure versetzt. Es bildet sich salzsaures Anilin, welches man mit Wasser auslaugt, während der 4-Dinitroindigo als dunkelblauer Niederschlag zurïckbleibt, der aus mikroskopisch kleinen, runden Körnern besteht. Dieser wird abfiltriert, mit Wasser gewaschen und getrocknet. Man erhält so den reinen 4-Dinitroindigo in dunkelblauen amorphen Stücken.

Die Analyse des bei $105^{\circ}$ getrockneten 4-Dinitroindigos ergab folgende Resultate:

$0.1701 g$ Substanz lieferten $0.3396 g$ Kohlensäure und $0.0367 g$ Wasser.

$0.1659 \mathrm{~g}$ Substanz gaben $23.6 \mathrm{~cm}^{3}$ Stickstoff bei $14^{\circ} \mathrm{C}$. und $750 \mathrm{~mm}$ Barometerstand.

Für die Molekulargewichtsbestimmung wurden bei Anwendung der kryoskopischen Methode von Baumann und From ${ }^{1}$ $0.1720 \mathrm{~g}$ 4-Dinitroindigo in $22 \mathrm{~g}$ Naphthalin gelöst. Die Depression betrug 0.15 Grade.

1 Berl. Ber., 24, 1432. 
In 100 Teilen:

\begin{tabular}{|c|c|c|}
\hline & $\begin{array}{l}\text { Berechnet fuir } \\
\mathrm{C}_{16} \mathrm{H}_{8} \mathrm{O}_{8} \mathrm{~N}_{4}\end{array}$ & Gefunden \\
\hline $\mathrm{C} \ldots \ldots$. & $54 \cdot 54$ & $54 \cdot 48$ \\
\hline $\mathrm{H}$ & $2 \cdot 27$ & $2 \cdot 40$ \\
\hline$N \ldots \ldots$ & $15 \cdot 91$ & $16 \cdot 35$ \\
\hline Mol.-Gew. . & 352 & $359 \cdot 7$ \\
\hline
\end{tabular}

4-Dinitroindigo ist unlöslich in Wasser, fast unlöslich in Salzsäure, Essigsäure, Alkohol, Äther, Ligroin und Benzol, wenig löslich in Aceton. In heißem Anilin löst er sich mit tief dunkelgrüner Farbe, in Phenol und Naphthalin mit dunkelblauer Farbe, ebenso in heißem Nitrobenzol zum Unterschiede vom Dinitroindigo, den Baeyer hergestellt hat, ${ }^{1}$ der sich in heißem Nitrobenzol mit kirschroter Farbe löst. Reduktionsmittel wie Zink und Salzsäure geben wahrscheinlich zuerst 4-Dinitroindigweiß, dann weiter 4-Diamidoindigweiß. An der Luft oxydiert sich dieses dann zu 4-Diamidoindigo, welcher zum Unterschiede von 4-Dinitroindigo in Essigsäure mit grüner Farbe löslich ist.

4-Dinitroindigo sublimiert, im Glasröhrchen erhitzt; auf dem Platinblech erhitzt, entwickelt er violette Dämpfe und verpufft schwach. Zerrieben zeigt 4-Dinitroindigo einen ähnlichen Metallglanz wie gewöhnlicher Indigo.

In Kürze zusammengefaßt, ergibt sich aus vorliegender Arbeit folgendes:

4-Nitro-2-Aminobenzoesäure gibt bei der Kondensation mit einem vierfachen Überschusse von Monochloressigsäure bei Gegenwart von Soda $m$-Nitrophenylglyzin-o-Carbonsäure, wobei zu beachten ist, daß, da bei der Reaktion sich Salzsäure bildet, während der ganzen Einwirkungsdauer die Flüssigkeit alkalisch bleiben muß. Außerdem bildet sich hiebei in kleinen Mengen infolge des großen Überschusses an Monochloressigsäure 5-Nitrophenyliminodiessigsäure-2-Carbonsäure, indem

I. Betl. Ber. 12, 1316. 
in der Amidogruppe der 4-Nitro-2-Aminobenzoesäure beide Wasserstoffatome durch Reste der Monochloressigsäure ersetzt werden.

Die $m$-Nitrophenylglyzin-o-Carbonsäure kondensiert sich mit Essigsäureanhydrid unter Austritt von Kohlensäure und Wasser zu 4-Nitrodiacetylindoxyl.

Dieses mit konzentrierter Schwefelsäure verseift, liefert, indem noch der Luftsauerstoff oxydierend einwirkt, 4-Dinitroindigo.

Zum Schlusse sei es mir noch gestattet, meinem hochverehrten Lehrer, Herrn Prof. Dr. Wilhelm Suida, für die Anregung und die erteilten Ratschläge meinen innigsten Dank auszusprechen. 